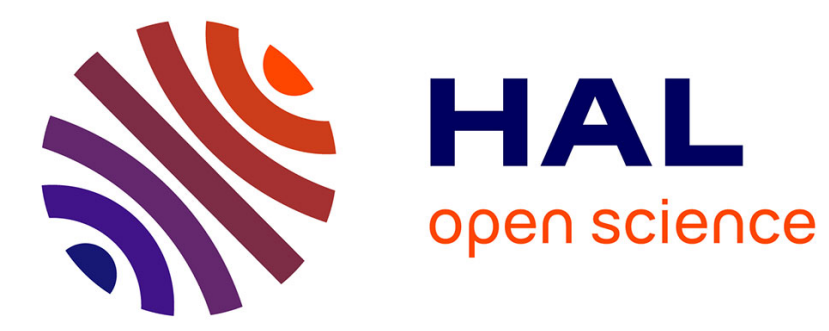

\title{
The Linear Constrained Control Problem for Discrete-Time Systems: Regulation on the Boundaries
}

\author{
George Bitsoris, Sorin Olaru, Marina Vassilaki
}

\section{To cite this version:}

George Bitsoris, Sorin Olaru, Marina Vassilaki. The Linear Constrained Control Problem for DiscreteTime Systems: Regulation on the Boundaries. Difference Equations, Discrete Dynamical Systems and Applications. ICDEA 2017., pp.215-245, 2019, 10.1007/978-3-030-20016-9_8 . hal-02908390

\section{HAL Id: hal-02908390 \\ https://hal-centralesupelec.archives-ouvertes.fr/hal-02908390}

Submitted on 29 Jul 2020

HAL is a multi-disciplinary open access archive for the deposit and dissemination of scientific research documents, whether they are published or not. The documents may come from teaching and research institutions in France or abroad, or from public or private research centers.
L'archive ouverte pluridisciplinaire HAL, est destinée au dépôt et à la diffusion de documents scientifiques de niveau recherche, publiés ou non, émanant des établissements d'enseignement et de recherche français ou étrangers, des laboratoires publics ou privés. 


\title{
The Linear Constrained Control Problem for Discrete-Time Systems: Regulation on the Boundaries
}

\author{
Georges Bitsoris, Sorin Olaru and Marina Vassilaki
}

\begin{abstract}
The chapter deals with the problem of regulation of linear systems around an equilibrium lying on the boundary of a polyhedral domain where linear constraints on the control and/or the state vectors are satisfied. In the first part of the chapter, the fundamental limitations for constrained control with active constraints at equilibrium are exposed. Next, based on the invariance properties of polyhedral and semi-ellipsoidal sets, design methods for guaranteeing convergence to the equilibrium while respecting linear control constraints are proposed. To this end, Lyapunov-like polyhedral functions, LMI methods and eigenstructure assignment techniques are applied.
\end{abstract}

\section{Introduction}

The regulation of linear systems by linear state-feedback under linear state and/or control constraints, called the Linear Constrained Regulation Problem (LCRP) [12], has been the object of intensive research work since the early publications on this subject [23], [31], [4], [5], [16]. The problem has been approached by applying optimization methods [31], [17], [29], eigenstructure assignment approaches [14] or Lyapunov methods [23], [4], [5], [16] using both quadratic [23] and polyhedral Lyapunov functions [31], [15].

\footnotetext{
G. Bitsoris

Control Systems Laboratory, Department of Electrical and Computer Engineering, University of Patras, 26500 Patras, Greece, e-mail: bitsoris@ece.upatras.gr

S. Olaru

Laboratory of Signals and Systems, CentraleSupelec, University Paris-Saclay Gif-sur-Yvette, 91190 France e-mail: sorin.olaru@ centralesupelec.fr
}

M. Vassilaki, Department of Electrical and Electronic Engineering Educators, ASPETE, 14121 Athens, Greece, e-mail: mvassilaki@aspete.gr 
An interpretation of the positive invariance in terms of geometric properties of some subspaces has also been given [24] and eigenstructure assignment techniques have been developed to solve other linear constrained regulation problems as the cases of linear systems with unbounded sets of state constraints [20]. Also, researches about linear dynamical systems admitting positively invariant cones have been developed and used for some control designs [19]. Since then, this approach has been used to solve related problems, as for instance: control in the presence of bounded persistent disturbances, control of uncertain systems, the LCRP with regional poleplacement, control by output feedback, among others. Furthermore, new approaches based on the use of quadratic Lyapunov functions have been developed for the determination of contractive ellipsoidal domains as estimates of the domain of attraction of the saturating closed-loop systems, mainly by applying LMI techniques. The vast literature on this subject is reviewed in [15].

In all these works, the desired equilibrium state is situated in the interior of the region where the state constraints are respected. In many engineering problems however, a regulation around an equilibrium lying on the boundary of this set is needed. This is always the case when the desired equilibrium state has to optimize a linear criterion or a norm in the state space. Such problems, for instance, are the regulation at least or maximal admissible velocity of a vehicle, the regulation on the upper admissible level of a tank system or the regulation at the nearest state from the boundary of an admissible region as in the case of obstacle avoidance problems where the goal is the regulation at an equilibrium situated as near as possible from a target point situated in the interior of a forbidden region. For this kind of problems the classical methods cannot be applied and only for some special cases design methods are available [19], [10]. The object of this chapter is to present the theoretical foundations and systematic design approaches to the LCRP concerning the regulation around an equilibrium situated on the boundary of the domain defined by the state constraints. For the analysis and design of this kind of control problems techniques based on the conditions of positive invariance of polyhedral sets, Lyapunov-like polyhedral functions, LMI approaches and eigenstructure assignment methods will be used.

The chapter is organized as follows: In Section 2, the notations adopted in this chapter and the problem statement are introduced. In Section 3, conditions guaranteeing the existence of a linear state-feedback control making the whole region defined by the state constraints an admissible domain of attraction are established. It is shown that if such a control exists then it can be determined by solving a linear programming problem. In the following sections, we investigate the case when a control resulting to the maximal admissible domain of attraction does not exists. Two particular cases are considered: in Section 4 we consider the case when the cone on which the equilibrium is situated can be positively invariant and in Section 5 the case when no linear state-feedback control making this cone positively invariant exists. For both cases, design techniques for the determination of a solution to the LCRP are proposed. 


\section{The linear constrained regulation problem}

Throughout this chapter, capital letters denote real matrices, lower case letters denote column vectors or scalars, $T$ denotes the time set $T=\{0,1,2, \ldots\}, \mathbb{R}^{n}$ denotes the real $n$-space, $\mathbb{R}_{+}^{p}\left(\mathbb{R}_{-}^{p}\right)$ is the nonnegative orthant (non positive orthant) of the real $p$-space, $\mathbb{R}^{n \times p}$ the set of real $n \times p$ matrices. $I_{p}$ denotes the $p \times p$ identity matrix, $0_{s \times q}$ denotes the $s \times p$ matrix with zero elements and $e_{p} \in \mathbb{R}^{p}$ is the vector

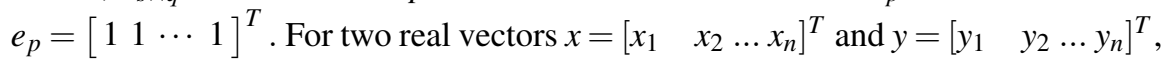
$x<y \quad(x \leq y)$ is equivalent to $x_{i}<y_{i} \quad\left(x_{i} \leq y_{i}\right) \quad i=1,2, \ldots, n$. Similar notation is applied for real matrices. For square matrices $P \in \mathbb{R}^{n \times n}, P \succ 0(P \succeq 0)$ means that $P$ is positive definite (positive semi-definite).

If $G \in \mathbb{R}^{s \times n}$ and $w \in \mathbb{R}^{s}$ then $\mathscr{P}\left(G, w_{x}\right)$ denotes the polyhedral set

$$
\mathscr{P}(G, w) \triangleq\left\{x \in \mathbb{R}^{n}: G x \leq w\right\}
$$

and $\mathscr{C}(G)$ denotes the polyhedral proper cone

$$
\mathscr{C}(G) \triangleq\left\{x \in \mathbb{R}^{n}: G x \leq 0\right\}
$$

In the case of a nonsingular square matrix $G \in \mathbb{R}^{n \times n}, \mathscr{C}(G)$ is said to be a simplicial proper cone.

Ellipsoidal sets are denoted by $\mathscr{Q}(G, d)$ and defined by the relation

$$
\mathscr{Q}(P, d) \triangleq\left\{x \in \mathbb{R}^{n}: x^{T} P x \leq d\right\}
$$

where $P \in \mathbb{R}^{n \times n}$ is a positive definite matrix and $d$ is a positive real number.

Finally, if $v(x)$ is a continuous function $v: \mathbb{R}^{n} \rightarrow \mathbb{R}_{+}$, and $d \in \mathbb{R}_{+}$, then $\mathscr{R}(v, d)$ denotes the set

$$
\mathscr{R}(v, d) \triangleq\left\{x \in \mathbb{R}^{n}: v(x) \leq d\right\}
$$

In this chapter, we consider linear discrete-time systems described by difference equations

$$
x(t+1)=A x(t)+B u(t)
$$

where $x \in \mathbb{R}^{n}$ is the state vector, $u \in \mathbb{R}^{m}$ is the input vector, $t \in T$ is the time variable and $A \in \mathbb{R}^{n \times n}, B \in \mathbb{R}^{n \times m}$.

The state vector is constrained to belong to a polyhedral set $\mathscr{P}\left(G, w_{x}\right)$ defined by the linear inequality

$$
G x \leq w_{x}
$$

where $G \in \mathbb{R}^{s \times n}$ and $w_{x} \in \mathbb{R}_{+}^{s}$.

The control input $u$ must also respect linear constraints of the form

$$
D u \leq \rho
$$

where $D \in \mathbb{R}^{q \times m}$ and $\rho \in \mathbb{R}^{q}$ is a vector with positive components. 
In this chapter we investigate the Linear Constrained Regulation Problem (LCRP) without a priori given domain of attraction [12]. This problem consists in the determination of a linear state feedback control law $u=F x$ and of a domain of attraction $\mathscr{D} \subseteq \mathscr{P}\left(G, w_{x}\right)$ such that for all initial states $x_{0} \in \mathscr{D}$ the corresponding trajectories $x\left(t ; x_{0}\right)$ of the resulting closed-loop system

$$
x(t+1)=(A+B F) x(t)
$$

converge to the equilibrium asymptotically while respecting the linear state and/or control constraints (2) and (3) respectively. Such a set $\mathscr{D}$ is said to be an admissible domain of attraction.

As it is the case for most of the works on constrained control, the positively invariant and linearly controlled invariant sets defined below play an important role in the investigation of the LCRP.

Definition 1: The subset $\mathscr{D} \subset \mathbb{R}^{n}$ of the state space of the autonomous system $x(t+1)=A x(t)$ is positively invariant if all trajectories $x\left(t ; x_{0}\right)$ starting from $\mathscr{D}$ remain in it, that is $x\left(t ; x_{0}\right) \in \mathscr{D}$ for all $x_{0} \in \mathscr{D}$ and $t \in T$.

Definition 2: The subset $\mathscr{D} \subset \mathbb{R}^{n}$ of the state space of system $x(t+1)=A x(t)+$ $B u(t)$ is linearly controlled invariant if there exists a linear state-feedback control $u=F x$ such that $\mathscr{D}$ is a positively invariant set of the resulting closed-loop system $x(t+1)=(A+B F) x(t)$.

In the following theorem, set conditions for a linear state feedback control law together with a subset $\mathscr{D} \subset \mathbb{R}^{n}$ to be a solution to the LCRP are given:

Theorem 1 ([12]): A control law $u=F x$ is a solution of the LCRP if and only if there exists a positively invariant set $\mathscr{D} \subset \mathbb{R}^{n}$ of the resulting closed-loop system (4) such that

a)

$$
\begin{aligned}
& \mathscr{D} \subseteq \mathscr{P}\left(G, w_{x}\right) \\
& \mathscr{D} \subseteq \mathscr{P}(D F, \rho)
\end{aligned}
$$

b) $\lim _{t \rightarrow \infty} x\left(t ; x_{0}\right)=0$ for all $x_{0} \in \mathscr{D}$.

Then, $\mathscr{D} \subset \mathbb{R}^{n}$ is an admissible domain of attraction.

The stabilizability of the pair $(A, B)$ is a necessary and sufficient condition for the existence of a solution to the LCRP if the origin is an interior point of set $\mathscr{P}\left(G, w_{x}\right)$, because any stabilizing linear state-feedback control together with a sufficiently small positively invariant set (e.g. an ellipsoidal set $\mathscr{D}=\mathscr{Q}(P, d)$ ) constitute a solution to the tis problem. Thus, in this case, the research interest is limited to the determination of the control law $u=F x$ which results to the maximal admissible domain of attraction $\mathscr{D}$, or/and to an admissible domain of attraction $\mathscr{D}$ with guaranteed performance [22], [15], [29], [27]. In the case, however, when the desired equilibrium $x_{e}=0$ is on the boundary of the set $\mathscr{P}\left(G, w_{x}\right)$, the stabilizability of the pair $(A, B)$ does not guarantee the existence of a solution to the LCRP and the design methods for the regulation around an interior point cannot be applied for the determination of such a solution. In this chapter, methods for solving the LCRP 
when the desired equilibrium $x_{e}=0$ is on a boundary hyperplane or on the vertex of a of the state constraints set $\mathscr{P}(G, w)$ are developed.

\section{Maximal admissible domains of attraction}

If the equilibrium state $x_{e}=0$ to be regulated is on the boundary of the set $\mathscr{P}(G, w)$ then at least one of the boundary hyperplanes $g_{j}^{T} x=w_{x j}$ of the set $\mathscr{P}\left(G, w_{x}\right)$ passes through the origin, therefore $w_{j}=0$. In order to simplify the notation, we assume that the equilibrium $x_{e}=0$ of the closed-loop system (4) is situated on the boundary hyperplanes

$$
g_{j}^{T} x=w_{x j} \quad j=1,2, \ldots, p \quad p<s
$$

Then

$$
\begin{gathered}
w_{x j}=0 \quad j=1,2, \ldots, p \\
w_{x j}>0 \quad j=p+1, p+2, \ldots, s
\end{gathered}
$$

and the inequality $G x \leq w_{x}$ which defines the polyhedral set $\mathscr{P}\left(G, w_{x}\right)$ is written as

$$
\begin{gathered}
G_{1} x \leq 0 \\
G_{2} x \leq w_{2}
\end{gathered}
$$

with

$$
\begin{gathered}
G_{1} \triangleq\left[\begin{array}{c}
g_{11}^{T} \\
g_{12}^{T} \\
\vdots \\
g_{1 p}^{T}
\end{array}\right]=\left[\begin{array}{c}
g_{1}^{T} \\
g_{2}^{T} \\
\vdots \\
g_{p}^{T}
\end{array}\right], G_{2} \triangleq\left[\begin{array}{c}
g_{21}^{T} \\
g_{22}^{T} \\
\vdots \\
g_{2 s}^{T}
\end{array}\right]=\left[\begin{array}{c}
g_{p+1}^{T} \\
g_{p+2}^{T} \\
\vdots \\
g_{s}^{T}
\end{array}\right], \\
w_{2} \triangleq\left[\begin{array}{c}
w_{21} \\
w_{22} \\
\vdots \\
w_{2(s-p)}
\end{array}\right]=\left[\begin{array}{c}
w_{x(p+1)} \\
w_{x(p+2)} \\
\vdots \\
w_{x s}
\end{array}\right]
\end{gathered}
$$

$g_{i}^{T} \quad i=1,2, \ldots, s$ being the rows of the matrix $G$.

Thus,

$$
\mathscr{P}\left(G, w_{x}\right)=\mathscr{C}\left(G_{1}\right) \cap \mathscr{P}\left(G_{2}, w_{2}\right)
$$

where $\mathscr{C}\left(G_{1}\right)$ denotes the polyhedral proper cone defined by inequality $G_{1} x \leq 0$. An illustration of this set is given in Figure 1.

We first investigate the case when there exists a linear state-feedback control making the whole region $\mathscr{P}\left(G, w_{x}\right)=\mathscr{C}\left(G_{1}\right) \cap \mathscr{P}\left(G_{2}, w_{2}\right)$ an admissible domain of attraction. This is the maximal admissible domain of attraction that can be established. According to Theorem 1, such a control law stabilizes the system and, in 


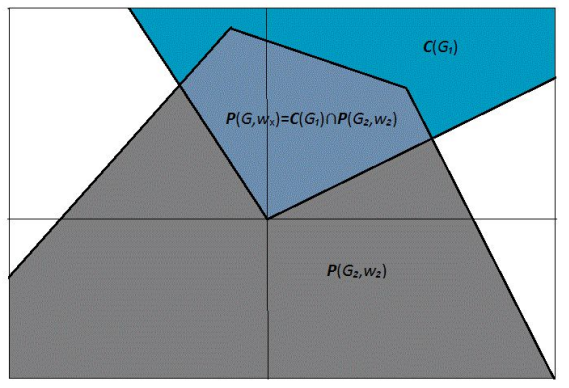

Fig. 1 Illustration of the composition of set $\mathscr{P}\left(G, w_{x}\right)$ as $\mathscr{P}\left(G, w_{x}\right)=\mathscr{C}\left(G_{1}\right) \cap \mathscr{P}\left(G_{2}, w_{2}\right)$

addition, makes the polyhedral set $\mathscr{P}\left(G, w_{x}\right)$ positively invariant with respect to the resulting closed-loop system (4). Conditions for the positive invariance of this set can be established by using the following result:

Lemma: The polyhedral set $\mathscr{P}(G, w)$ with $G \in \mathbb{R}^{s \times n}$ and $w \in \mathbb{R}_{+}^{s \times n}$ is positively invariant with respect to system $x(t+1)=A x(t)$ if and only if there exists a matrix $H \in \mathbb{R}^{s \times s}$, such that

$$
\begin{gathered}
G A=H G \\
H \geq 0 \\
H w \leq w
\end{gathered}
$$

This result is a special case of a more general result on the positive invariance of sets defined by vector inequalities $v(x) \leq w$ with respect to iterated systems defined in partially ordered spaces [11]. Conditions for the special classes of polyhedral sets having the origin as an interior point with respect to linear discrete-time systems have been initially established by Bitsoris ([7],[9]) for bounded or unbounded polyhedral sets and by Molchanov and Pyatninskii [25], Benjaouia and Burgat [4] and Blanchini [16] for polytopes.

Using the result stated in the preceding lemma, we can establish necessary and sufficient conditions for the positive invariance of polyhedral sets of the form $\mathscr{P}\left(G, w_{x}\right)=\mathscr{C}\left(G_{1}\right) \cap \mathscr{P}\left(G_{2}, w_{2}\right)$.

Theorem 2: The set $\mathscr{P}\left(G, w_{x}\right)=\mathscr{C}\left(G_{1}\right) \cap \mathscr{P}\left(G_{2}, w_{2}\right)$ is positively invariant with respect to system (4) if and only if there exist matrices $H_{11} \in \mathbb{R}^{p \times p}, H_{21} \in \mathbb{R}^{(s-p) \times p}$ and $H_{22} \in \mathbb{R}^{(s-p) \times(s-p)}$ satisfying the relations

$$
\begin{gathered}
G_{1}(A+B F)=H_{11} G_{1} \\
H_{11} \geq 0
\end{gathered}
$$


Title Suppressed Due to Excessive Length

$$
\begin{gathered}
G_{2}(A+B F)=H_{21} G_{1}+H_{22} G_{2} \\
H_{21} \geq 0, H_{22} \geq 0 \\
H_{22} w_{2} \leq w_{2}
\end{gathered}
$$

Proof: By virtue of Lemma 1, the set $\mathscr{P}\left(G, w_{x}\right)$ is positively invariant with respect to system (4) if and only if there exist nonnegative matrices $H_{11} \in \mathbb{R}^{p \times p}, H_{12} \in$ $\mathbb{R}^{p \times(s-p)}, H_{21} \in \mathbb{R}^{(s-p) \times p}$ and $H_{22} \in \mathbb{R}^{(s-p) \times(s-p)}$ such that

$$
\begin{gathered}
G_{1}(A+B F)=H_{11} G_{1}+H_{12} G_{2} \\
G_{2}(A+B F)=H_{21} G_{1}+H_{22} G_{2} \\
H_{11} \cdot 0+H_{12} w_{2} \leq 0 \\
H_{21} \cdot 0+H_{22} w_{2} \leq w_{2}
\end{gathered}
$$

Since $w_{2}>0$ and $H_{12} \geq 0$, inequality (14) is satisfied if and only if $H_{12}=0$. Thus, conditions (12)-(15) are equivalent to relations (7)-(11). $\square$

Remark 1: The positive invariance of the proper cone $\mathscr{C}\left(G_{1}\right)$ of the polyhedral set $\mathscr{P}\left(G, w_{x}\right)=\mathscr{C}\left(G_{1}\right) \cap \mathscr{P}\left(G_{2}, w_{2}\right)$ is a necessary condition for the positive invariance of the polyhedral set $\mathscr{P}\left(G, w_{x}\right)$ with respect to system (4). This is expressed by conditions (7) and (8) of Theorem 2. If, in addition, $r a n k G_{1}=n$ and the nonnegative matrix $H_{11}$ has all its eigenvalues in the interior of the unit disk of the complex plane, then any gain matrix $F$ that satisfies condition (7) is the gain matrix of a stabilizing control law $u=F x$ for system (1).

Remark 2: If $x_{0} \in \mathscr{P}\left(G, w_{x}\right)$ then $G_{1} x_{0} \leq 0$ and $G_{2} x_{0} \leq w_{2}$. Therefore, from conditions (9)-(11) it follows that

$$
\begin{aligned}
G_{2}(A+B F) x_{0} & =H_{21} G_{1} x_{0}+H_{22} G_{2} x_{0} \\
& \leq H_{22} G_{2} x_{0} \\
& \leq w_{2}
\end{aligned}
$$

which means that $x_{0} \in \mathscr{C}\left(G_{1}\right) \cap \mathscr{P}\left(G_{2}, w_{2}\right)$ implies $x\left(t ; x_{0}\right) \in \mathscr{P}\left(G_{2}, w_{2}\right)$ for all $t \in T$. These conditions, however, do not guarantee the positive invariance of the polyhedral set $\mathscr{P}\left(G_{2}, w_{2}\right)$. Therefore, the positive invariance of the polyhedral subset $\mathscr{P}\left(G_{2}, w_{2}\right)$ is not a necessary condition of the positive invariance of the set $\mathscr{P}\left(G, w_{x}\right)=\mathscr{C}\left(G_{1}\right) \cap \mathscr{P}\left(G_{2}, w_{2}\right)$

We are now in a position to establish conditions for a bounded set $\mathscr{P}\left(G, w_{x}\right)=$ $\mathscr{C}\left(G_{1}\right) \cap \mathscr{P}\left(G_{2}, w_{2}\right)$ to be an admissible domain of attraction.

Theorem 3: If the set $\mathscr{C}\left(G_{1}\right) \cap \mathscr{P}\left(G_{2}, w_{x 2}\right)$ is bounded and for a matrix $F \in$ $\mathbb{R}^{m \times n}$ there exist a real number $\varepsilon$ and matrices $H_{11} \in \mathbb{R}^{p \times p}, H_{21} \in \mathbb{R}^{(s-p) \times p}, H_{22} \in$ $\mathbb{R}^{(s-p) \times(s-p)}$ and $L \in \mathbb{R}^{q \times s}$ satisfying relations (7)-(10) and

$$
\begin{gathered}
H_{22} w_{2} \leq \varepsilon w_{2} \\
\varepsilon<1
\end{gathered}
$$




$$
\begin{gathered}
L\left[\begin{array}{l}
G_{1} \\
G_{2}
\end{array}\right]=D F \\
L\left[\begin{array}{c}
0 \\
w_{2}
\end{array}\right] \leq \rho \\
L \geq 0
\end{gathered}
$$

then the set $\mathscr{P}\left(G, w_{x}\right)=\mathscr{C}\left(G_{1}\right) \cap \mathscr{P}\left(G_{2}, w_{2}\right)$ is an admissible domain of attraction of the resulting closed-loop system (4).

Proof:

a) Sufficiency: By virtue of Theorem 2, conditions (7)-(10) and (16)-(17) imply the positive invariance of the set $\mathscr{D}=\mathscr{C}\left(G_{1}\right) \cap \mathscr{P}\left(G_{2}, w_{2}\right)$. Moreover, by virtue of Farkas lemma, conditions (18)-(20) are equivalent to the set relation $\mathscr{P}\left(G_{1}, 0\right) \cap$ $\mathscr{P}\left(G_{2}, w_{2}\right) \subseteq \mathscr{P}(D F, \rho)$ or, equivalently, to the set relation $\mathscr{C}\left(G_{1}\right) \cap \mathscr{P}\left(G_{2}, w_{2}\right) \subseteq$ $\mathscr{P}(D F, \rho)$. To complete the proof, we shall prove that $u=F x$ is a stabilizing control in $\mathscr{P}\left(G, w_{x}\right)=\mathscr{C}\left(G_{1}\right) \cap \mathscr{P}\left(G_{2}, w_{2}\right)$.

Let $v(x)$ be the continuous function defined by relation

$$
v(x) \triangleq \max _{1 \leq i \leq s-p}\left\{\frac{\left(G_{2} x\right)_{i}}{w_{2 i}}\right\} \triangleq \max _{p+1 \leq i \leq s}\left\{\frac{g_{i}^{T} x}{w_{2 i}}\right\}
$$

The function $v(x)$ is positive definite in $\mathscr{C}\left(G_{1}\right) \cap \mathscr{P}\left(G_{2}, w_{2}\right)$, that is $v(0)=0$ and $v(x)>0$ for all $x \in \mathscr{C}\left(G_{1}\right) \cap \mathscr{P}\left(G_{2}, w_{2}\right)$ and $x \neq 0$. The second property holds because otherwise there would exist a $x \in \mathscr{C}\left(G_{1}\right) \cap \mathscr{P}\left(G_{2}, w_{2}\right), x \neq 0$ such that $G_{2} x \leq 0$. Then for any $r>0$ it would follow that

$$
\begin{gathered}
G_{1}(r x) \leq 0 \\
G_{2}(r x) \leq 0<w_{2}
\end{gathered}
$$

which would contradict the hypothesis that set $\mathscr{C}\left(G_{1}\right) \cap \mathscr{P}\left(G_{2}, w_{2}\right)$ is bounded. Furthermore, if $x \in \mathscr{C}\left(G_{1}\right) \cap \mathscr{P}\left(G_{2}, w_{2}\right)$, that is if $G_{1} x \leq 0$ and $G_{2} x \leq w_{2}$, then from (9)-(10) and (16)-(17) it follows that

$$
\begin{aligned}
v[(A+B F) x] & =\max _{1 \leq i \leq s-p}\left\{\frac{\left(G_{2}(A+B F) x\right)_{i}}{w_{2 i}}\right\} \\
& =\max _{1 \leq i \leq s-p}\left\{\frac{\left(\left(H_{21} G_{1}+H_{22} G_{2}\right) x\right)_{i}}{w_{2 i}}\right\} \\
& =\max _{1 \leq i \leq s-p}\left\{\frac{\left(H_{22} v(x) w_{2}\right)_{i}}{w_{2 i}}\right\} \\
& \leq \max \left\{\varepsilon \frac{w_{2 i}}{w_{2 i}}\right\} v(x) \\
& \leq \varepsilon v(x)
\end{aligned}
$$

because $G_{2} x \leq v(x) w_{2}$ Thus, $v[(A+B F) x]-v(x)$ is negative definite in $\mathscr{C}\left(G_{1}\right) \cap$ $\mathscr{P}\left(G_{2}, w_{2}\right)$ because, by (17), $\varepsilon<1$. Therefore, $\lim _{t \rightarrow \infty} x\left(t ; x_{0}\right)=0$ for all $x_{0} \in \mathscr{C}\left(G_{1}\right) \cap$ 
$\mathscr{P}\left(G_{2}, w_{2}\right)$ because the function $v(x)$ is continuous. Thus, all the hypotheses of Theorem 1 are satisfied. Consequently, the set $\mathscr{C}\left(G_{1}\right) \cap \mathscr{P}\left(G_{2}, w_{2}\right)$ is an admissible domain of attraction.

b)Necessity:According to Theorem 1, for the control law $u=F x$ to render the bounded set $\mathscr{D}=C\left(G_{1}\right) \cap \mathscr{P}\left(G_{2}, w_{2}\right)$ an admissible domain of attraction for system

(4) it is necessary that

a) $\mathscr{D}$ is positively invariant

b) $\mathscr{D} \subseteq \mathscr{P}\left(D F, \rho_{u}\right)$

c) $\mathscr{D}$ is a domain of attraction.

By Theorem 2, for the set $\mathscr{D}=C\left(G_{1}\right) \cap \mathscr{P}\left(G_{2}, w_{2}\right)$ to be positively invariant, it is necessary that conditions (7)-(10) and (16) are satisfied for a $\varepsilon \leq 1$. On the other hand, by virtue of Farkas Lemma, for $\mathscr{D} \subseteq \mathscr{P}\left(D F, \rho_{u}\right)$ to be satisfied it is necessary that there exists a nonnegative matrix $L$ satisfying relations (18) and (19).

Remark 3: The scalar function $v(x)$ defined by (21) cannot be viewed as a Lyapunov function in the state space of the system because it is not positive definite in $\mathbb{R}^{n}$. This function, however, expresses the distance $d(x, 0)$ of a state $x \in C\left(G_{1}\right) \cap \mathscr{P}\left(G_{2}, w_{2}\right)$ from the origin, if the distance $d(x, y)$ in this bounded set is defined by the relation

$$
d(x, y)=\left\{\begin{array}{cc}
0 & \text { if } x=y \\
v(x)+v(y) & \text { if } x \neq y
\end{array}\right.
$$

The application of this result to the determination of a control law $u=F x$ resulting to a maximal admissible domain of attraction $\mathscr{D}=\mathscr{C}\left(G_{1}\right) \cap \mathscr{P}\left(G_{2}, w_{2}\right)$ is straightforward: such a control law is obtained by solving the linear programming problem

$$
\min _{H_{11}, H_{21}, H_{22}, L, F, \varepsilon}\{\varepsilon\}
$$

under constraints (7)-(10), (16) and (18)-(20).

If $\arg \min \{\varepsilon\}<1$ and the set $\mathscr{C}\left(G_{1}\right) \cap \mathscr{P}\left(G_{2}, w_{2}\right)$ is bounded, then the so obtained control $u=F x$ is a stabilizing one and $\mathscr{C}\left(G_{1}\right) \cap \mathscr{P}\left(G_{2}, w_{2}\right)$ is an admissible domain of attraction. This is also true in the case when the set $\mathscr{C}\left(G_{1}\right) \cap \mathscr{P}\left(G_{2}, w_{2}\right)$ is unbounded provided that the resulting closed-loop matrix $A+B F$ has all its eigenvalues in the interior of the unit disk of the complex plane. In both cases, due to (22), the so obtained control law provides the greatest rate of convergence if the distance from the origin of a state $x \in \mathscr{C}\left(G_{1}\right) \cap \mathscr{P}\left(G_{2}, w_{2}\right)$ is measured by the scalar function $v(x)$ defined by (21).

If the result of the optimization problem (23) under constraints (7)-(10) and (16)-(20) is $\hat{\varepsilon}=1$ then, with the corresponding optimal control $u=\hat{F} x$, the set $\mathscr{P}\left(G, w_{x}\right)=\mathscr{C}\left(G_{1}\right) \cap \mathscr{P}\left(G_{2}, w_{2}\right)$ is positively invariant. This set is also an admissible domain of attraction if, in addition, all eigenvalues of matrix $A+B F$ are in the interior of the unit disk of the complex plane.

Example 1: We consider a second order discrete-time linear system $x(t+1)=$ $A x(t)+b u(t)$ with 


$$
A=\left[\begin{array}{cc}
0.25 & 0.25 \\
-0.5 & 1
\end{array}\right], b=\left[\begin{array}{l}
0.5 \\
-1
\end{array}\right]
$$

and linear state constraints $G x \leq w_{x}$ where

$$
G=\left[\begin{array}{cc}
-0.9 & 0.5 \\
-0.95 & -0.14 \\
1 & 0.25 \\
0.07 & -0.53
\end{array}\right], \quad w_{x}=\left[\begin{array}{l}
0 \\
1 \\
1 \\
1
\end{array}\right]
$$

The control input $u$ is constrained to satisfy the linear inequality $D u \leq \rho$ where

$$
D=\left[\begin{array}{c}
10 \\
-1
\end{array}\right], \rho=\left[\begin{array}{l}
0.2 \\
0.6
\end{array}\right]
$$

It is cleat that the origin is situated on the plane (degenerated cone) defined by the inequality

$$
-0.9 x_{1}+0.5 x_{2} \leq 0
$$

Solving the linear programming problem (23) under constraints (7)-(10) and (16)(20) with

$$
G_{1}=\left[\begin{array}{ll}
-0.9 & 0.5
\end{array}\right], \quad G_{2}=\left[\begin{array}{cc}
-0.95 & -0.14 \\
1 & 0.25 \\
0.07 & -0.53
\end{array}\right], \quad w_{2}=\left[\begin{array}{l}
1 \\
1 \\
1
\end{array}\right]
$$

we determine the optimal values

$$
\hat{\varepsilon}=0.934 \quad \hat{F}=[-0.2450 .1478]
$$

Since the set $\mathscr{P}\left(G, w_{x}\right)$ is bounded and $\hat{\varepsilon}<1$, by virtue of Theorem 3, with the control law

$$
u=-0.245 x_{1}+0.1478 x_{2}
$$

the origin is an asymptotically stable equilibrium of the resulting closed-loop system (4) and the set $\mathscr{P}\left(G, w_{x}\right)$ is an admissible domain of attraction. $\square$

Finally, if this optimization problem is not feasible or it is feasible but $\arg \min \{\varepsilon\}>$ 1 then there does not exist any linear state-feedback control law making the set $\mathscr{P}\left(G, w_{x}\right)=\mathscr{C}\left(G_{1}\right) \cap \mathscr{P}\left(G_{2}, w_{2}\right)$ positively invariant and, as a result, neither an admissible domain of attraction. This means that the set $\mathscr{P}\left(G, w_{x}\right)$ cannot be an admissible domain of attraction. In these cases, an admissible domain of attraction will necessarily be a strict subset of the polyhedral set $\mathscr{P}\left(G, w_{x}\right)=\mathscr{C}\left(G_{1}\right) \cap \mathscr{P}\left(G_{2}, w_{2}\right)$, provided that the LCRP has a solution. These cases are investigated in the following sections of the chapter. 
Title Suppressed Due to Excessive Length

4 Domains of attraction of the form $\mathscr{D}=\mathscr{C}\left(G_{1}\right) \cap \mathscr{D}_{2}$

We first consider the case when the maximal set $\mathscr{C}\left(G_{1}\right) \cap \mathscr{P}\left(G_{2}, w_{2}\right)$ cannot be a positively invariant set, and as a result nor an admissible domain of attraction, but a stabilizing control $u=F x$ rendering the cone $\mathscr{C}\left(G_{1}\right)$ positively invariant exists. Then a set of the form $\mathscr{D}=\mathscr{C}\left(G_{1}\right) \cap \mathscr{D}_{2}$ may be an admissible domain of attraction.

In the following theorem, we establish conditions for a linear state-feedback control to stabilize the system while rendering the cone $\mathscr{C}\left(G_{1}\right)$ positively invariant:

Theorem 4: The linear state-feedback control $u=F x$ stabilizes the system (1) and renders the cone $\mathscr{C}\left(G_{1}\right)$ positively invariant if and only if there exist three matrices $H_{11} \in \mathbb{R}^{p \times p}, Q \in \mathbb{R}^{n \times n}$ and $Y \in \mathbb{R}^{m \times n}$, satisfying the relations

$$
\begin{gathered}
G_{1} A Q+G_{1} B Y=H_{11} G_{1} Q \\
H_{11} \geq 0 \\
{\left[\begin{array}{cc}
Q & A Q+B Y \\
(A Q+B Y)^{T} & Q
\end{array}\right] \succ 0}
\end{gathered}
$$

Proof:

a) Sufficiency: If relation (27) is satisfied, then $Q \succ 0$ and consequently $Q$ is nonsingular. Thus, setting

$$
F=Y Q^{-1}
$$

from (25) it follows that

$$
G_{1}(A+B F)=H_{11} G_{1}
$$

which, together with (26), imply the positive invariance of the cone $\mathscr{C}\left(G_{1}\right)$ with respect to the closed-loop system $x(t+1)=(A+B F) x(t)$. Moreover, from (27) it follows that the matrix $Q$ is positive definite and

$$
Q-(A Q+B Y)^{T} Q^{-1}(A Q+B Y) \succ 0
$$

or, equivalently,

$$
Q-Q\left(A+B Y Q^{-1}\right)^{T} Q^{-1}\left(A+B Y Q^{-1}\right) Q \succ 0
$$

or, finally,

$$
Q^{-1}-\left(A+B Y Q^{-1}\right)^{T} Q^{-1}\left(A+B Y Q^{-1}\right) \succ 0
$$

because the matrix $Q^{-1}$ is also positive definite. Now, taking into account (28), this relation is equivalently written as

$$
Q^{-1}-(A+B F)^{T} Q^{-1}(A+B F) \succ 0
$$

which means that $v(x)=x^{T} Q^{-1} x$ is a Lyapunov function for the system $x(t+1)=$ $(A+B F) x(t)$. Therefore $F=Y Q^{-1}$ is the gain matrix of a stabilizing linear statefeedback control for system (1). 
b) Necessity: If there exists a stabilizing control $u=F x$ then there also exists a symmetric positive definite matrix $P$ that satisfies the Lyapunov matrix inequality

$$
P-(A+B F)^{T} P(A+B F) \succ 0
$$

Since matrix $P$ is positive definite their inverse exists and is also symmetric and positive definite. Therefore, there exists a matrix $Y$ such that $F=Y P$. Thus, relation (30) is written as

$$
P-(A+B Y P)^{T} P(A+B Y P) \succ 0
$$

or, equivalently,

$$
P-P^{-1}\left(A P^{-1}+B Y\right)^{T} P\left(A P^{-1}+B Y\right) P \succ 0
$$

or, finally,

$$
P^{-1}-\left(A P^{-1}+B Y\right)^{T} P\left(A P^{-1}+B Y\right) \succ 0
$$

Setting $Q=P^{-1}$ we obtain

$$
Q-(A Q+B Y)^{T} Q^{-1}(A Q+B Y) \succ 0
$$

which, by virtue of the Schur complement theorem, is equivalent to condition (27).

If, in addition, the control law $u=Y P x=Y Q^{-1} x$ renders the cone $\mathscr{C}\left(G_{1}\right)$ positively invariant, then, by virtue of Theorem 2 , there exists a nonnegative matrix $H_{11}$ such that

$$
G_{1}(A+B F)=H_{11} G_{1}
$$

or

$$
G_{1}(A+B Y P)=H_{11} G_{1}
$$

or, finally,

$$
G_{1}(A Q+B Y)=H_{11} G_{1} Q
$$

A solution to the LCRP can be obtained by first solving relations (25), (27) to determine a stabilizing control $u=Y Q^{-1} x$ rendering the cone $\mathscr{C}\left(G_{1}\right)$ positively invariant and then by determining an admissible domain of attraction $\mathscr{D}$ of the form

$$
\mathscr{D}=\mathscr{C}\left(G_{1}\right) \cap \mathscr{D}_{2}
$$

where $\mathscr{D}_{2}$ is positively invariant set of the resulting closed-loop system such that

$$
\mathscr{C}\left(G_{1}\right) \cap \mathscr{D}_{2} \subset \mathscr{C}\left(G_{1}\right) \cap \mathscr{P}\left(G_{2}, w_{2}\right)
$$

and

$$
\mathscr{C}\left(G_{1}\right) \cap \mathscr{D}_{2} \subseteq P(D F, \rho)
$$

This can be done by constructing a quadratic Lyapunov function $v(x)=x^{T} P x$ for the closed-loop system and then by determining the maximal value of the positive 
constant $d$ so that the set $\mathscr{D}_{2}=\mathscr{Q}(P, d)$ satisfies set relations (31) and , (32). To this end, one can use the quadratic Lyapunov function $v(x)=x^{T} Q^{-1} x, Q$ being the matrix obtained by solving relations (25), (27) to determine the stabilizing control $u=Y Q^{-1} x$. Any other quadratic Lyapunov function may also be used.

Admissible domains of attraction can also be obtained by simply determining a polyhedral positively invariant set $\mathscr{P}\left(G_{2}^{*}, w_{2}^{*}\right)$ for the resulting closed-loop system such that

$$
\mathscr{C}\left(G_{1}\right) \cap \mathscr{P}\left(G_{2}^{*}, w_{2}^{*}\right) \subset \mathscr{C}\left(G_{1}\right) \cap \mathscr{P}\left(G_{2}, w_{2}\right)
$$

and

$$
\mathscr{C}\left(G_{1}\right) \cap \mathscr{P}\left(G_{2}^{*}, w_{2}^{*}\right) \subseteq P(D F, \rho)
$$

These approaches, however, require the determination of a solution of the nonlinear relations (25), (27). In the following subsection, we show how this difficult nonlinear problem can be solved in the cases when the equilibrium is situated on one boundary hyperplane of the state constraint set $\mathscr{P}\left(G, w_{x}\right)$ or on the vertex of a proper cone.

\subsection{Equilibrium on one boundary hyperplane}

In the case when only one boundary hyperplane of the polyhedral set $\mathscr{P}\left(G, w_{x}\right)=$ $\mathscr{C}\left(G_{1}\right) \cap \mathscr{P}\left(G_{2}, w_{2}\right)$ passes through the origin, the matrix $G_{1}$ is a line vector $g_{1}^{T}$ and thus $\mathscr{C}\left(G_{1}\right)$ degenerates to a half space defined by relation $g_{1}^{T} x \leq 0$. Then, the necessary and sufficient conditions (25)-(27) for the existence of a stabilizing control $u=Y Q^{-1} x$ rendering the half-space $g_{1}^{T} x \leq 0$ positively invariant become

$$
\begin{gathered}
g_{1}^{T} A Q+g_{1}^{T} B Y=h_{1} g_{1}^{T} Q \\
{\left[\begin{array}{cc}
Q & A Q+B Y \\
(A Q+B Y)^{T} & Q
\end{array}\right] \succ 0} \\
h_{1} \geq 0
\end{gathered}
$$

Conditions (33) and (35) express the fact that $g_{1}^{T}$ is a left eigenvector of matrix $A+B Y Q^{-1}=A+B F$ associated with a nonnegative eigenvalue $h_{1}$. Therefore, there exists an admissible domain of attraction of the form $\mathscr{C}\left(g_{1}^{T}\right) \cap D_{2}$ if and only if there exists a stabilizing control that assigns $g_{1}^{T}$ as a left eigenvector of the matrix $A+B F$. associated with eigenvalue $h_{1}$ such that

$$
0 \leq h_{1}<1
$$

Consequently, if there exists a stabilizing control $u=F x$ that assigns $g_{1}^{T}$ as a left eigenvector of matrix $A+B F$, then its gain matrix $F=Y Q^{-1}$ can be determined by solving the parametrized convex problem (33) and (35) with the scalar parameter $h_{1}$ belonging to the interval $[0,1)$. 
Having computed a stabilizing control making the half-space $\mathscr{C}\left(g_{1}^{T}\right)$ positively invariant, the next step is the determination of an admissible domain of attraction. Two approaches are proposed:

\subsubsection{Semi-ellipsoidal domains of attraction}

As already mentioned, having computed a stabilizing control making the half-space $\mathscr{C}\left(g_{1}^{T}\right)$, if $v(x)=x^{T} P x$ is a Lyapunov function of the resulting closed-loop system, then any set $\mathscr{D}=\mathscr{C}\left(g_{1}^{T}\right) \cap \mathscr{Q}(P, d)$ satisfying the set relations

$$
\mathscr{C}\left(g_{1}^{T}\right) \cap \mathscr{Q}(P, d) \subset \mathscr{C}\left(g_{1}^{T}\right) \cap \mathscr{P}\left(G_{2}, w_{2}\right)
$$

and

$$
\mathscr{C}\left(g_{1}^{T}\right) \cap \mathscr{Q}(P, d) \subseteq \mathscr{P}(D F, \rho)
$$

These relations are satisfied if

$$
\mathscr{Q}(P, d) \subset \mathscr{P}\left(G_{2}, w_{2}\right)
$$

and

$$
\mathscr{Q}(P, d) \subseteq \mathscr{P}(D F, \rho)
$$

or equivalently [18] if

$$
d g_{2 i}^{T} P^{-1} g_{2 i} \leq w_{2 i} \quad i=1,2, \ldots, s-p
$$

and

$$
d(D F)_{i}^{T} P^{-1}(D F)_{i} \leq \rho_{i} \quad i=1,2, \ldots q
$$

Thus, by determining the maximal value $\hat{d}$ of parameter $d$ satisfying inequalities (37) and (38) we determine the maximal hyperellipsoid $\mathscr{Q}(P, d)$ included in the sets $\mathscr{P}\left(G_{2}, w_{2}\right)$ and $\mathscr{P}(D F, \rho)$. Since all sets $\mathscr{Q}(P, d)$ for $d>0$ are attractive, the set $\mathscr{D}=\mathscr{C}\left(g_{1}^{T}\right) \cap \mathscr{Q}(P, \hat{d})$ is an admissible domain of attraction.

Example 2: We consider a discrete-time linear system $x(t+1)=A x(t)+b u(t)$ with matrices

$$
A=\left[\begin{array}{cc}
-0.8 & 0.3 \\
1.6 & 0
\end{array}\right], \quad b=\left[\begin{array}{c}
-0.5 \\
2
\end{array}\right]
$$

and control constraints $D u \leq \rho$ where

$$
D=\left[\begin{array}{c}
-1 \\
0.5
\end{array}\right], \quad \rho=\left[\begin{array}{l}
1 \\
1
\end{array}\right]
$$

The state must satisfy linear constraints $G x \leq w_{x}$ with 


$$
G=\left[\begin{array}{cc}
-0.9 & 0.5 \\
-1 & -0.25 \\
0.5 & -0.1667 \\
1.0 & 0.25
\end{array}\right], \quad w_{x}=\left[\begin{array}{l}
0 \\
1 \\
1 \\
1
\end{array}\right]
$$

The polyhedral set $\mathscr{P}\left(G, w_{x}\right)$ can be written as $\mathscr{P}\left(G, w_{x}\right)=\mathscr{C}\left(g_{1}^{T}\right) \cap \mathscr{P}\left(G_{2}, w_{2}\right)$ where

$$
\begin{gathered}
g_{1}^{T}=\left[\begin{array}{ll}
-0.9 & 0.5
\end{array}\right] \\
G_{2}=\left[\begin{array}{cc}
-1 & -0.25 \\
0.5 & -0.1667 \\
1.0 & 0.25
\end{array}\right], \quad w_{2}=\left[\begin{array}{l}
1 \\
1 \\
1
\end{array}\right]
\end{gathered}
$$

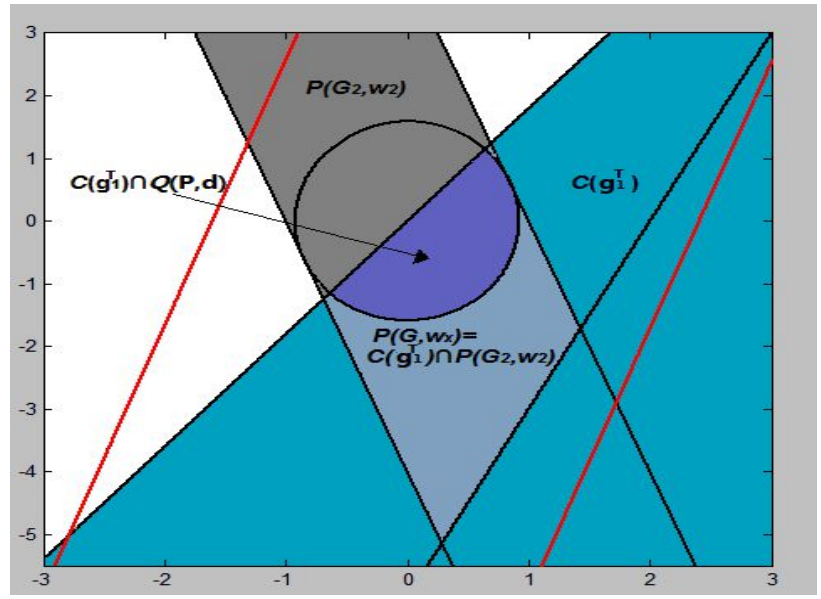

Fig. 2 The half-space $C\left(g_{1}^{T}\right)$, the unbounded polyhedral set $P\left(G_{2}, w_{2}\right)$ and the semi-ellipsoidal admissible set $C\left(g_{1}^{T}\right) \cap Q(P, d) \subset C\left(g_{1}^{T}\right) \cap P\left(G_{2}, w_{2}\right)$ of the Example 2 .

It is clear that the origin is situated on the edge $g_{1}^{T} x=0$ of the polyhedron $\mathscr{P}\left(G, w_{x}\right)$. In can be easily verified that the linear programming problem (23) under constraints (7)-(10) and (16)-(20) has no solution. Therefore, there does not exist any linear state-feedback control rendering the maximal set $\mathscr{C}\left(g_{1}^{T}\right) \cap \mathscr{P}\left(G_{2}, w_{2}\right)$ an admissible domain of attraction. Since, as it can be easily seen, there exists a control law $u=F x$ such that $g_{1}^{T}$ is a left eigenvector of the closed-loop matrix $A+b F$ origin we shall derive a stabilizing linear state-feedback control $u=F x$ making a set of the form $\mathscr{C}\left(g_{1}^{T}\right) \cap \mathscr{Q}(P, d)$ an admissible domain of attraction set of the resulting closed-loop system. To this end, we solve the parametrized convex problem (33) and (35) with the scalar parameter $h_{1}$ belonging to the interval $[0,1)$. The optimal values of the unknown parameters are

$$
\hat{Q}=\left[\begin{array}{ll}
0.2242 & 0.0996 \\
0.0996 & 0.4694
\end{array}\right], \quad \hat{Y}=\left[\begin{array}{ll}
-0.2502 & 0.0136
\end{array}\right]
$$




$$
\hat{h}_{1}=0.33 \quad \hat{F}=\hat{Y} \hat{Q}^{-1}=\left[\begin{array}{ll}
-1.2463 & 0.2935
\end{array}\right]
$$

Since $\hat{h}_{1}<1$,

$$
u=\hat{F} x=-1.2463 x_{1}+0.2935 x_{2}
$$

is a stabilizing control law. Finally, we determine the maximal value $\hat{d}=3.297$ of the parameter $d$ that satisfies the inequalities (37)-(38). Thus, the set $\mathscr{C}\left(g_{1}^{T}\right) \cap$ $\mathscr{Q}\left(Q^{-1}, \hat{d}\right)$ is an admissible domain of attraction of the resulting closed-loop system.

\subsubsection{Polyhedral domains of attraction}

The second approach consists in determining a polyhedral admissible domain of attraction of the form $\mathscr{D}=\mathscr{C}\left(g_{1}^{T}\right) \cap \mathscr{P}\left(G_{2}^{*}, w_{2}^{*}\right)$, that is a domain of attraction which is the intersection of the half-space $\mathscr{C}\left(g_{1}^{T}\right)$ and of a polyhedral set $\mathscr{P}\left(G_{2}^{*}, w_{2}^{*}\right)$. We can determine a polyhedral positively invariant set $\mathscr{P}\left(G_{2}^{*}, e_{p *}\right), G_{2}^{*} \in \mathbb{R}^{p * n}$ for the resulting asymptotically stable system (4), by applying one of the well known methods of construction of polyhedral positively invariant sets for stable linear systems [8],[24],[29]. Since all polyhedral sets $\mathscr{P}\left(G_{2}^{*}, r e_{p *}\right)$ with $r>0$, obtained by scaling the set $\mathscr{P}\left(G_{2}^{*}, e_{p *}\right)$, are also positively invariant, by virtue of Theorem 1 , for constructing an admissible domain of attraction it is sufficient to determine a $r$ such that

$$
\begin{gathered}
\mathscr{C}\left(g_{1}^{T}\right) \cap \mathscr{P}\left(G_{2}^{*}, r e_{p *}\right) \subset \mathscr{C}\left(g_{1}^{T}\right) \cap \mathscr{P}\left(G_{2}, w_{2}\right) \\
\mathscr{C}\left(g_{1}^{T}\right) \cap \mathscr{P}\left(G_{2}^{*}, r e_{p *}\right) \subseteq \mathscr{P}(D F, \rho) .
\end{gathered}
$$

To this end, we can use the following result:

Theorem 5: The set relations (39) and (40) are satisfied if and only if there exist matrices $K_{1} \in \mathbb{R}^{(s-p) \times p}, K_{2} \in \mathbb{R}^{(s-p) \times p *}$ and $L \in \mathbb{R}^{q \times(1+p *)}$. such that

$$
\begin{gathered}
K_{1} g_{1}^{T}+K_{2} G_{2}^{*}=G_{2} \\
r K_{2} e_{p *} \leq w_{2} \\
L\left[\begin{array}{c}
g_{1}^{T} \\
G_{2}^{*}
\end{array}\right]=D F \\
L\left[\begin{array}{c}
0 \\
r e_{p *}
\end{array}\right] \leq \rho \\
L \geq 0, K_{i} \geq 0 \quad i=1,2
\end{gathered}
$$

Proof: The set relations (39) and (40) are equivalently written as

$$
\begin{gathered}
{\left[\begin{array}{l}
g_{1}^{T} \\
G_{2}^{*}
\end{array}\right] x \leq\left[\begin{array}{c}
0 \\
r e_{p *}
\end{array}\right] \Rightarrow\left[\begin{array}{l}
g_{1}^{T} \\
G_{2}
\end{array}\right] x \leq\left[\begin{array}{c}
0 \\
w_{2}
\end{array}\right]} \\
{\left[\begin{array}{l}
g_{1}^{T} \\
G_{2}^{*}
\end{array}\right] x \leq\left[\begin{array}{c}
0 \\
r e_{p *}
\end{array}\right] \Rightarrow D F x \leq \rho}
\end{gathered}
$$


By Farkas Lemma, these relations are satisfied if and only there exist nonnegative real matrices $M \in \mathbb{R}^{s \times(1+p *)}$ and $L \in \mathbb{R}^{q \times(1+p *)}$ such that

$$
\begin{gathered}
M\left[\begin{array}{l}
g_{1}^{T} \\
G_{2}^{*}
\end{array}\right]=\left[\begin{array}{l}
g_{1}^{T} \\
G_{2}
\end{array}\right] \text { and } M\left[\begin{array}{c}
0 \\
r e_{p *}
\end{array}\right] \leq\left[\begin{array}{c}
0 \\
w_{2}
\end{array}\right] \\
L\left[\begin{array}{l}
g_{1}^{T} \\
G_{2}^{*}
\end{array}\right]=D F \text { and } L\left[\begin{array}{c}
0 \\
r e_{p *}
\end{array}\right] \leq \rho
\end{gathered}
$$

Partitioning matrix $M$ as follows

$$
M=\left[\begin{array}{ll}
M_{1} & M_{2} \\
M_{3} & M_{4}
\end{array}\right]
$$

with $M_{1} \in \mathbb{R}, M_{2} \in \mathbb{R}^{1 \times p *}, M_{3} \in \mathbb{R}^{p \times p}$ and $M_{4} \in \mathbb{R}^{p \times p *}$, relations (46) are equivalently written as

$$
\begin{gathered}
M_{1} g_{1}^{T}+M_{2} G_{2}^{*}=g_{11}^{T} \\
M_{3} g_{1}^{T}+M_{4} G_{2}^{*}=G_{2} \\
r M_{2} e_{p *} \leq 0 \\
r M_{4} e_{p *} \leq w_{2}
\end{gathered}
$$

These relations are satisfied for

$$
M_{2}=0, M_{1}=I
$$

and

$$
\begin{gathered}
M_{3} g_{1}^{T}+M_{4} G_{2}^{*}=G_{2} \\
r M_{4} e_{p *} \leq w_{2}
\end{gathered}
$$

Thus, setting $K_{1}=M_{3}$ and $K_{2}=M_{4}$, we obtain conditions (41) and (42). $\square$

According to this theorem, starting from a positively invariant set $\mathscr{P}\left(G_{2}^{*}, e_{p *}\right)$ for the resulting closed-loop system, we construct an admissible polyhedral domain of attraction $\mathscr{P}\left(G_{2}^{*}, r e_{p *}\right)=\mathscr{P}\left(G_{2}^{*}, \hat{r}^{-1} e_{p *}\right)$ by solving the linear programming problem

$$
\min _{L, K_{1}, K_{2}, \hat{r}}\{\hat{r}\}
$$

under constraints

$$
\begin{gathered}
K_{1} g_{1}^{T}+K_{2} G_{2}^{*}=G_{2} \\
K_{2} e_{p *} \leq \hat{r} w_{2} \\
L\left[\begin{array}{l}
g_{1}^{T} \\
G_{2}^{*}
\end{array}\right]=D F
\end{gathered}
$$




$$
\begin{gathered}
L\left[\begin{array}{c}
0 \\
e_{p *}
\end{array}\right] \leq \hat{r} \rho \\
K_{i} \geq 0 \quad i=1,2, \quad L \geq 0,
\end{gathered}
$$

It is clear that the so obtained admissible domain $\mathscr{D}=\mathscr{C}\left(g_{1}^{T}\right) \cap \mathscr{P}\left(G_{2}^{*}, r e_{p *}\right)$ is not unique because an asymptotically stable linear system possesses many positively invariant polyhedral sets $\mathscr{P}\left(G_{2}^{*}, e_{p *}\right)$. It is however possible to enlarge an initially determined admissible domain of attraction not by scaling but using techniques of determination of maximal positively invariant sets [22], [27] or by applying the recently established approach of enlargement of positively invariant sets with specified complexity [2], [3].

Example 3: We consider again the discrete-time linear system $x(t+1)=A x(t)+$ $b u(t)$ with matrices $A$ and $b$ given by (24) with the same control constraints but with state constraints $G x \leq w$ with

$$
G=\left[\begin{array}{cc}
-0.8821 & 0.4712 \\
-1 & -0.2500 \\
0.5 & -0.1667
\end{array}\right], \quad w=\left[\begin{array}{l}
0 \\
1 \\
1
\end{array}\right]
$$

The control input $u$ has also to satisfy the linear constraints $D u \leq \rho$ where

$$
D=\left[\begin{array}{c}
10 \\
-1
\end{array}\right], \rho=\left[\begin{array}{l}
0.2 \\
0.6
\end{array}\right]
$$

Solving the parametrized convex problem (33)- (35) with

$$
\begin{gathered}
G_{1}=\left[\begin{array}{ll}
-0.90 & 0.50
\end{array}\right], \quad G_{2}=\left[\begin{array}{cc}
-0.95 & -0.14 \\
1 & 0.25 \\
0.07 & -0.53
\end{array}\right], w_{2}=\left[\begin{array}{l}
1 \\
1 \\
1
\end{array}\right] \\
D=\left[\begin{array}{c}
10 \\
-1
\end{array}\right], \rho=\left[\begin{array}{l}
0.2 \\
0.6
\end{array}\right]
\end{gathered}
$$

we obtained the optimal values

$$
\begin{gathered}
Q==\left[\begin{array}{cc}
-0.95 & -0.14 \\
1 & 0.25-0.53
\end{array}\right], \quad Y=\left[\begin{array}{ll}
-0.245 & 0.1478
\end{array}\right] \\
\hat{\varepsilon}=0.934 \quad \hat{F}=Y Q^{-1}=[-0.2450 .1478]
\end{gathered}
$$

Since $\hat{\varepsilon}<1$ and the set $\mathscr{P}(G, w)$ is bounded, by virtue of Theorem 3, with the control law

$$
u=-0.245 x_{1}+0.1478 x_{2}
$$

the origin is an asymptotically stable equilibrium of the resulting closed-loop system (4) and the set $\mathscr{P}(G, w)$ is an admissible domain of attraction. $\square$ 


\subsection{Equilibrium on the vertex of a proper cone}

We consider now the case when $G_{1} \in \mathbb{R}^{p \times n}$ and $\operatorname{rank} G_{1}=n$. In this case, $p$, with $p \geq n$, boundary hyperplanes of the set $\mathscr{P}\left(G, w_{x}\right)=\mathscr{C}\left(G_{1}\right) \cap \mathscr{P}\left(G_{2}, w_{2}\right)$ pass through the origin and $\mathscr{C}\left(G_{1}\right)$ is a proper cone. For the existence of an admissible domain of attraction of the form $\mathscr{D}=\mathscr{C}\left(G_{1}\right) \cap D_{2}$ it is necessary and sufficient that there exists a stabilizing control law $u=F x$ making the proper cone $\mathscr{C}\left(G_{1}\right)$ positively invariant with respect to the resulting closed-loop system (4).

A control $u=F x$ makes the proper cone $\mathscr{C}\left(G_{1}\right)$ with $G_{1} \in \mathbb{R}^{p \times n}$ and $\operatorname{rank} G_{1}=n$ a positively invariant set of the closed-loop system $x(t+1)=(A+B F) x(t)$ if and only if there exists a matrix $H_{11} \in \mathbb{R}^{p \times p}$ such that

$$
\begin{gathered}
G_{1} A+G_{1} B F=H_{11} G_{1} \\
H_{11} \geq 0
\end{gathered}
$$

The control $u=F x$ is, in addition, a stabilizing one, if all the eigenvalues of the resulting closed-loop matrix $A+B F$ are in the interior of the unit disk of the complex plane. If $(\lambda, v)$ is an eigenpair of matrix $A+B F$ then $\lambda G_{1} v=G_{1}(A+B F) v=$ $H_{11} G_{1} v$, that is $\left(\lambda, G_{1} v\right)$ is an eigenpair of matrix system $H_{11}$. Moreover, since all the elements of matrix $H_{11}$ are nonnegative, if $0 \leq \lambda<1$ and $G v>0$, then all the eigenvalues of matrix $H_{11}$ are in the interior of the unit disk of the complex plane because all its elements are nonnegative [21]. This, in turn, implies that all the eigenvalues of the closed-loop matrix $A+B F$ are also in the interior of the unit disk of the complex plane as a consequence of the assumption that $\operatorname{rank}_{1}=n$. Therefore, a control law $u=F x$ assigning a positive eigenvalue $\lambda<1$ with an associated eigenvector $v, G_{1} v>0$ and satisfying relation (54) for a nonnegative matrix $H_{11}$, is a stabilizing control law that makes the proper cone $\mathscr{C}\left(G_{1}\right)$ positively invariant.

Since the simultaneous assignment of an arbitrary eigenvalue and an eigenvector is not always possible, the determination of the control law $u=F x$ may be achieved by replacing condition $H_{11} G_{1} v=\lambda G_{1} v$ by the inequality $H_{11} G_{1} v \leq \lambda G_{1} v$, because in this case also the eigenvalues of matrix $H_{11}$ are in the open unit disk of the complex plane [21]. Thus, the determination of such a control law $u=F x$ may be achieved by solving the linear programming problem

$$
\min _{F, H_{11}, \varepsilon}\{\varepsilon\}
$$

under constraints

$$
\begin{aligned}
G_{1} A+G_{1} B F & =H_{11} G_{1} \\
H_{11} w^{*} & \leq \varepsilon w^{*} \\
H_{11} & \geq 0
\end{aligned}
$$

where $w^{*} \in \mathbb{R}^{p}$ is an arbitrarily chosen vector with positive components belonging to the range of matrix $G_{1}$. If $\arg \min \{\varepsilon\}<1$ then the control $u=F x$ stabilizes the system and makes the cone $\mathscr{C}\left(G_{1}\right)$ positively invariant. 
Using this result we can establish an approach for the determination of a stabilizing state-feedback control and of an admissible domain of attraction of the form $D=\mathscr{C}\left(G_{1}\right) \cap \mathscr{D}_{2}$. The first step is the determination of a stabilizing control $u=F x$ making the cone $\mathscr{C}\left(G_{1}\right)$ positively invariant. This can be done by solving the linear programming problem (56)-(59). Next, we apply one of the methods established in subsections 4.1.1 and 4.1.2 for determining a semi-ellipsoidal $\mathscr{P}\left(G, w_{x}\right)=\mathscr{C}\left(G_{1}\right) \cap \mathscr{Q}\left(P_{2}, d_{2}\right)$ or a polyhedral $\mathscr{P}\left(G, w_{x}\right)=\mathscr{C}\left(G_{1}\right) \cap \mathscr{P}\left(G_{2}^{*}, w_{2}\right)$ admissible domain of attraction.

\section{Domains of attraction of the form $\mathscr{D}=\mathscr{C}\left(G_{1}^{*}\right) \cap \mathscr{D}_{2}$, $\mathscr{C}\left(G_{1}^{*}\right) \subset \mathscr{C}\left(G_{1}\right)$}

We consider now the case when there does not exist any stabilizing gain matrix $F$ and nonnegative matrix $H_{11}$ satisfying condition $G_{1}(A+B F)=H_{11} G_{1}$. This means that the cone $\mathscr{C}\left(G_{1}\right)$ cannot be positively invariant and thus its faces cannot be boundary hyperplanes of an admissible domain of attraction. It is clear that a "quadratic" approach consisting in the determination of a paraboloidal positively invariant set $\mathscr{R}(v, 0) \subset \mathscr{C}\left(G_{1}\right)$ with $\mathscr{R}(v, 0)$ being a set defined by a second order polynomial inequality $v(x) \leq 0$ where

$$
v(x)=x^{T} P x+l^{T} x
$$

is naturally excluded if $\mathscr{C}\left(G_{1}\right)$ is a proper cone. We shall show that it is also excluded in the case when the cone $\mathscr{C}\left(G_{1}\right)$ is degenerated to a half-space defined by an inequality $g_{1}^{T} x \leq 0$. This follows from the following theorem:

Theorem 6: If there exist a matrix $F$ and a function $v(x), v: \mathbb{R}^{n} \rightarrow \mathbb{R}$

$$
v(x)=x^{T} P x+l^{T} x
$$

such that the set $\mathscr{R}(v, 0)$ is positively invariant with respect to. the resulting closedloop system and satisfies the set relation $\mathscr{R}(v, 0) \subset \mathscr{C}\left(g_{1}^{T}\right)$ then the cone $\mathscr{C}\left(g_{1}^{T}\right)$ is also positively invariant.

Proof:

If there exists a control $u=F x$ making a set of the form

$$
\mathscr{R}(v, 0)=\left\{x \in \mathbb{R}^{n}: x^{T} P x+l^{T} x \leq 0\right\}
$$

positively invariant with respect to the closed-loop system $\dot{x}=(A+B F) x$ and such that

$$
\mathscr{R}(v, 0) \subset \mathscr{C}\left(G_{1}\right)
$$

then the surface $v(x)=0$ is tangent to the hyperplane $g_{1}^{T} x=0$ at the point $x=0$, that is

$$
\left.\nabla v(x)\right|_{x=0}=r g_{1}
$$

for a $r>0$. Therefore, 


$$
l=r g_{1}
$$

and

$$
v(x)=x^{T} P x+r g_{1}^{T} x
$$

Furthermore, if the set $R(v, 0)$ is positively invariant then

$$
v[(A+B F) x] \leq v(x)
$$

or

$$
x^{T}(A+B F)^{T} P(A+B F) x+r g_{1}^{T}(A+B F) x \leq x^{T} P x+r g_{1}^{T} x
$$

for all $x \in \mathbb{R}^{n}$ such that $v(x) \leq d$, or equivalently for all $x$ satisfying the inequality

$$
x^{T} P x+\operatorname{rg}_{1}^{T} x \leq d
$$

For this implication to be satisfied for $x$ belonging to a neighborhood of the origin, it is necessary that

$$
\begin{gathered}
r g_{1}^{T}(A+B F) x \leq r g_{1}^{T} x \\
r g_{1}^{T} x \leq 0
\end{gathered}
$$

which is equivalent to the existence of a nonnegative real number $\hat{h}$ such that

$$
\hat{h} r g_{1}^{T}=r g_{1}^{T}(A+B F)
$$

This, however, implies that the degenerated cone $\mathscr{C}\left(g_{1}^{T}\right)$ is positively invariant .

According to this result, in the case when there does not exist any control law making the half-space $g_{1}^{T} x \leq 0$ positively invariant, then a natural candidate admissible domain of attraction will be of the form $\mathscr{D}=\mathscr{C}\left(G_{1}^{*}\right) \cap \mathscr{D}_{2}$ where $\mathscr{C}\left(G_{1}^{*}\right)$ is a polyhedral cone such that $\mathscr{C}\left(G_{1}^{*}\right) \subseteq \mathscr{C}\left(G_{1}\right)$.

For a set $\mathscr{D}=\mathscr{C}\left(G_{1}^{*}\right) \cap \mathscr{D}_{2}$ to be an admissible domain of attraction it is necessary that the cone $\mathscr{C}\left(G_{1}^{*}\right)$ is positively invariant and $\mathscr{C}\left(G_{1}^{*}\right) \subseteq \mathscr{C}\left(G_{1}\right)$. This is equivalent to the existence of two matrices $H_{11}^{*} \in \mathbb{R}^{n \times n}$ and $L \in \mathbb{R}^{n \times n}$ such that

$$
\begin{gathered}
G_{1}^{*}(A+B F)=H_{11}^{*} G_{1}^{*} \\
H_{11}^{*} \geq 0 \\
L G_{1}^{*}=G_{1} \\
L \geq 0
\end{gathered}
$$

Relations (61) and (62) guarantee the positive invariance of the proper cone $\mathscr{C}\left(G_{1}^{*}\right)$ and relations (63) and (64) are equivalent to the set relation $\mathscr{C}\left(G_{1}^{*}\right) \subseteq \mathscr{C}\left(G_{1}\right)$.

The determination of a gain matrix $F$ and of a cone $\mathscr{C}\left(G_{1}^{*}\right)$ with $G_{1}^{*}$ satisfying relations (61)-(64) is a nonlinear problem which, however, for some special but important cases can be solved by convenient eigenstructure assignment approaches. In the following subsections, the two important cases when only one boundary hyper- 
plane and when $n$ boundary hyperplanes of the set $\mathscr{C}\left(G_{1}\right) \cap \mathscr{P}\left(G_{2}, w_{2}\right)$ pass through the origin are considered.

\subsection{Equilibrium on one boundary hyperplane}

As already mentioned, when only one boundary hyperplane of set $\mathscr{C}\left(G_{1}\right) \cap \mathscr{P}\left(G_{2}, w_{2}\right)$ passes through the origin, the cone $\mathscr{C}\left(G_{1}\right)$ is degenerated to a half-space defined by the relation $g_{1}^{T} x \leq 0$.

If the pair $(A, B)$ is controllable then, by applying an eigenvalue assignment approach, we can determine a gain matrix $F$ such that all eigenvalues $\lambda_{i} \quad i=1,2, \ldots, n$ of matrix $A+B F$ are distinct and $0 \leq \lambda_{i}<1$. Each eigenvalue is associated with a real left eigenvector $g_{1 i}^{* T} \quad i=1,2, \ldots, n$. The signs of the linearly independent associated left eigenvectors $g_{1 i}^{* T} \quad i=1,2, \ldots, n$ can be chosen so that a nonnegative vector $k \in \mathbb{R}^{n}, k=\left[\begin{array}{llll}k_{1} & k_{2} & \cdots & k_{n}\end{array}\right]^{T}$ satisfying the relation

$$
k_{1} g_{11}^{* T}+k_{2} g_{21}^{* T}+\ldots+k_{n} g_{1 n}^{* T}=g_{1}^{T}
$$

can be determined. Then, setting

$$
G_{1}^{*}=\left[\begin{array}{c}
g_{11}^{* T} \\
g_{12}^{* T} \\
\vdots \\
g_{1 n}^{* T}
\end{array}\right]
$$

we get

$$
G_{1}^{*}(A+B F)=H_{11}^{*} G_{1}^{*}
$$

and

$$
k^{T} G_{1}^{*}=g_{1}^{T}
$$

where $H_{11}^{*}$ is the nonnegative matrix $H_{11}^{*}=\operatorname{diag}\left(\lambda_{1}, \lambda_{2}, \ldots, \lambda_{n}\right)$. Relation (65) together with $H_{11}^{*} \geq 0$ guarantee the positive invariance of the cone $\mathscr{C}\left(G_{1}^{*}\right)$ and relation (66) together with $k \geq 0$ imply that $\mathscr{C}\left(G_{1}^{*}\right) \subset \mathscr{C}\left(g_{1}^{T}\right)$. Moreover, from (65) it follows that $u=F x$ is a stabilizing control because, by construction, the matrix $H_{11}^{*}$ has stable eigenvalues and $r a n k G_{1}^{*}=n$.

The next step is the determination of a subset $\mathscr{D}_{2}^{*} \subseteq \mathscr{P}\left(G_{2}, w_{2}\right)$ such that $\mathscr{D}=\mathscr{C}\left(G_{1}^{*}\right) \cap \mathscr{D}_{2}^{*}$ is positively invariant and $\mathscr{C}\left(G_{1}^{*}\right) \cap \mathscr{D}_{2}^{*} \subset \mathscr{P}(D F, \rho)$. To this end, one first examine whether $\mathscr{D}=\mathscr{C}\left(G_{1}^{*}\right) \cap \mathscr{P}\left(G_{2}, w_{2}\right)$ is an admissible domain of attraction. This can be done by solving the linear programming problem

$$
\min _{H_{21}, H_{22}, L, \varepsilon}\{\varepsilon\}
$$

under constraints 
Title Suppressed Due to Excessive Length

$$
\begin{gathered}
G_{2}(A+B F)=H_{21} G_{1}^{*}+H_{22} G_{2} \\
H_{22} w_{2} \leq \varepsilon w_{2} \\
H_{21} \geq 0, \quad H_{22} \geq 0 \\
L\left[\begin{array}{c}
G_{1}^{*} \\
G_{2}
\end{array}\right]=D F \\
L\left[\begin{array}{c}
0 \\
w_{2}
\end{array}\right] \leq \rho \\
L \geq 0
\end{gathered}
$$

If the optimal value $\hat{\varepsilon}$ satisfies the inequality $\hat{\varepsilon} \leq 1$ then $\mathscr{D}=\mathscr{C}\left(G_{1}^{*}\right) \cap \mathscr{P}\left(G_{2}, w_{2}\right)$ is an admissible domain of attraction. Otherwise, by applying one of the approaches established in the subsections 4.1.1 and 4.1.2, a domain of attraction $\mathscr{D}=\mathscr{C}\left(G_{1}^{*}\right) \cap$ $\mathscr{D}_{2}^{*}$ with $\mathscr{C}\left(G_{1}^{*}\right) \cap \mathscr{D}_{2}^{*} \subseteq \mathscr{C}\left(G_{1}^{*}\right) \cap \mathscr{P}\left(G_{2}, w_{2}\right)$ can be determined.

\subsection{Equilibrium on the vertex of a proper cone}

Next, we consider the case when $\mathscr{C}\left(G_{1}\right)$ is a proper cone, that is when $G_{1} \in \mathbb{R}^{n \times n}$ and $\operatorname{det} G_{1} \neq 0$. If there does not exist any stabilizing linear control making the cone $\mathscr{C}\left(G_{1}\right)$ positively invariant, then we search for a linear control making a set of the form $\mathscr{C}\left(G_{1}^{*}\right) \cap \mathscr{D}_{2}$ an admissible domain of attraction, where $\mathscr{C}\left(G_{1}^{*}\right)$ is a proper cone such that $\mathscr{C}\left(G_{1}^{*}\right) \subset \mathscr{C}\left(G_{1}\right)$. A method for determining a stabilizing control $u=F x$ rendering a proper cone $\mathscr{C}\left(G_{1}^{*}\right), \mathscr{C}\left(G_{1}^{*}\right) \subset \mathscr{C}\left(G_{1}\right)$ positively invariant can be developed using the following result:

Theorem 7: If there exist positive real numbers $\lambda_{i}^{*} \quad i=1,2, \ldots, n$, a nonnegative matrix $L^{*} \in \mathbb{R}^{n \times n}$ and matrices $V^{*} \in \mathbb{R}^{n \times n}$ and $Y \in \mathbb{R}^{m \times n}$, with $\operatorname{det} V^{*} \neq 0$ such that

$$
\begin{gathered}
A V^{*}+B Y=V^{*} \operatorname{diag}\left(\lambda_{1}^{*}, \lambda_{2}^{*}, \ldots, \lambda_{n}^{*}\right) \\
0 \leq \lambda_{i}^{*}<1 \quad i=1,2, \ldots, n \\
L^{*}=G_{1} V^{*}
\end{gathered}
$$

then, with the state feedback control $u=F x$ where

$$
F=Y V^{*-1},
$$

the equilibrium $x_{e}=0$ of the resulting closed-loop system (4) is asymptotically stable, the proper cone $\mathscr{C}\left(V^{*-1}\right)$ is positively invariant and $\mathscr{C}\left(V^{*-1}\right) \subseteq \mathscr{C}\left(G_{1}\right)$.

Proof:

From relations (74) and (77) it follows that

$$
A V^{*}+B F V^{*}=V^{*} \operatorname{diag}\left(\lambda_{1}^{*}, \lambda_{2}^{*}, \ldots, \lambda_{n}^{*}\right)
$$


and taking into account that $\operatorname{det} V^{*} \neq 0$ we get

$$
V^{*-1}(A+B F)=\operatorname{diag}\left(\lambda_{1}^{*}, \lambda_{2}^{*}, \ldots, \lambda_{n}^{*}\right) V^{*-1}
$$

Since the matrix $H_{1} \triangleq \operatorname{diag}\left(\lambda_{1}^{*}, \lambda_{2}^{*}, \ldots, \lambda_{n}^{*}\right)$ is nonnegative, from (78) it follows that the cone $\mathscr{C}\left(V^{*-1}\right)$ is positively invariant with respect to system (4). Furthermore, $u=F x$ is a stabilizing control because $\lambda_{i}^{*} i=1,2, \ldots, n$ are the eigenvalues of matrix $A+B F$ and $0 \leq \lambda_{i}^{*}<1$. Finally, taking into account that $L^{*} \geq 0$, from relation (76) which can written as $L^{*} V^{*-1}=G_{1}$, it follows that $\mathscr{C}\left(V^{*-1}\right) \subseteq \mathscr{C}\left(G_{1}\right)$.

Using this result we can develop eigenstructure assignment approaches for determining a stabilizing linear state feedback control together with a positively invariant cone included in $\mathscr{C}\left(G_{1}\right)$.

From

$$
(A+B F) V^{*}=V^{*} \operatorname{diag}\left(\lambda_{1}^{*}, \lambda_{2}^{*}, \ldots, \lambda_{n}^{*}\right)
$$

which is equivalent to (74) it follows that the columns $v_{i}^{*} i=1,2, \ldots, n$ of matrix $V_{1}^{*}$ are the eigenvectors of the closed-loop matrix $A+B F$ associated with the eigenvalues $\lambda_{i}^{*} i=1,2, \ldots, n$. Thus, taking into account relations (74)-(76) and $L^{*} \geq 0$, we conclude that for determining a stabilizing linear state feedback control and a positively invariant cone $\mathscr{C}\left(G_{1}^{*}\right)$ included in $\mathscr{C}\left(G_{1}\right)$ one have to derive a gain matrix $F$ assigning the eigenvalues of the closed-loop system in the interval $[0,1)$ with corresponding eigenvectors $v_{i}$ satisfying the inequalities

$$
G_{1} v_{i}^{*} \geq 0
$$

It should be noticed that the existence of a solution to this eigenstructure assignment problem is not guaranteed even if the pair $(A, B)$ is controllable [28], [1]. Sufficient conditions of existence of such a solution may be established using the results on the spectral characterization of systems possessing positively invariant cones [30] •

The final step is the determination of a subset $\mathscr{D}_{2}^{*} \subseteq \mathscr{P}\left(G_{2}, w_{2}\right)$ such that $\mathscr{D}=\mathscr{C}\left(V_{1}^{*-1}\right) \cap \mathscr{D}_{2}^{*}$ is positively invariant and $\mathscr{C}\left(V_{1}^{*-1}\right) \cap \mathscr{D}_{2}^{*} \subset \mathscr{P}(D F, \rho)$. This can be done by applying one of the approaches established in the subsections 4.1.1 and 4.1.2.

Example 4: We consider a discrete-time linear system $x(t+1)=A x(t)+b u(t)$ with

$$
A=\left[\begin{array}{cr}
0 & 1.4 \\
2.2 & -2
\end{array}\right], b=\left[\begin{array}{l}
0.5 \\
1.5
\end{array}\right]
$$

The control vector is constrained to satisfy the inequalities

$$
u \leq \rho_{1}
$$

with $\rho_{1}=10$ and $\rho_{2}=-$ It is also given a bounded polyhedral subset $\mathscr{P}\left(G, w_{x}\right)$ of system's state space where 


$$
G=\left[\begin{array}{cc}
-0.2 & 1 \\
-1.2 & 0.4 \\
-0.5 & -0.5
\end{array}\right], \quad w_{x}=\left[\begin{array}{l}
1 \\
1 \\
1
\end{array}\right]
$$

The set $\mathscr{P}\left(G, w_{x}\right)$ represents a forbidden region in which for safety reasons the trajectories of the system must not enter. Thus, the admissible region of evolution of the state vector is the non-convex set $\bar{P}=\mathbb{R}^{n} \backslash \operatorname{Int} \mathscr{P}\left(G, w_{x}\right)$. The control objective is the determination of a control law and of a subset $\mathscr{D} \subset \mathscr{P}$ such that all trajectories of the resulting closed-loop system emanating from the set $\mathscr{D}$ are transferred asymptotically to an equilibrium $x_{e}$ lying as close as possible to the origin.

The states that can be equilibria satisfy the relation $A x_{e}+b u_{e}=x_{e}$, for a steady state control $u_{e}$. This implies that the admissible equilibrium states are situated on the line $1.3 x_{e 1}-1.8 x_{e 2}=0$. By adopting the function $v(x)=\max _{1 \leq i \leq 3}\left\{\frac{g_{i}^{T} x}{w_{x i}}\right\}$ as a distance of the state $x$ from the origin, the admissible equilibrium states $x_{e}$ are also situated on the boundary of the forbidden region $\mathscr{P}\left(G, w_{x}\right)$. Thus, with $u_{e}^{1}=-0.04255$ and $u_{e}^{2}=0.02439$ we obtain the two admissible equilibrium states

$$
x_{e}^{1}=\left[\begin{array}{c}
1.91489 \\
1.3829
\end{array}\right], x_{e}^{2}=\left[\begin{array}{l}
-1.0975 \\
-0.7926
\end{array}\right]
$$

situated, respectively, on the boundary faces $-0.2 x_{1}+x_{2}=1$ and $-1.2 x_{1}+0.4 x_{2}=$ 1 of the forbidden region $\mathscr{P}\left(G, w_{x}\right)$.

We first consider the equilibrium $x_{e}^{1}$ lying on the boundary line $-0.2 x_{1}+x_{2}=1$ and we examine whether there exists a stabilizing control $u(k)=u_{e}^{1}+f^{i^{T}}\left[x(k)-x_{e}^{1}\right]$ making the half space $-0.2 x_{1}+x_{2} \geq 1$ positively invariant w.r.t. the resulting closedloop system $x(k+1)=A x(k)+b f^{i}\left[x(k)-x_{e}^{i}\right]+b u_{e}^{1}$. Setting $z(k)=x(k)-x_{e}^{1}$, this problem is converted to a problem of determination of a stabilizing gain vector $f^{i} \in$ $\mathbb{R}^{2}$ for the system

$$
z(k+1)=\left(A+b f^{i^{T}}\right) z(k)
$$

making the half space $0.2 z_{1}-z_{2} \leq 0$ positively invariant. To this end, we solve the parametrized convex problems (33)-(35) with $g_{1}^{T}=[0.2-1]$ and we obtain

$$
\begin{gathered}
h_{1}=0.35 \\
Q=\left[\begin{array}{cc}
8.9622 & -11.2756 \\
-11.2756 & 23.5890
\end{array}\right] \\
Y=\left[\begin{array}{ll}
-35.7136 & 62.5965
\end{array}\right]
\end{gathered}
$$

Thus, with the control $u(t)=f^{1^{T}}\left(x(k)-x_{e}^{1}\right)+u_{e}^{1}$ where $f^{1^{T}}=Y Q^{-1}=[-1.62141 .8786]$ the equilibrium $x_{e}^{1}$ of system $x(t+1)=A x(t)+b u(t)$ becomes asymptotically stable and the half space $-0.2 x_{1}+x_{2} \geq 1$ is positively invariant. Then any set $\mathscr{D}_{1}=\mathscr{P}\left(-g_{1}^{T},-1\right) \cap \mathscr{Q}_{x-x_{e}^{1}}\left(Q^{-1}, d\right)$ where

$$
\mathscr{Q}_{x-x_{e}^{1}}\left(Q^{-1}, d\right)=\left\{x \in \mathbb{R}^{2}:\left(x-x_{e}^{1}\right)^{T} Q^{-1}\left(x-x_{e}^{1}\right) \leq r\right\}
$$


with $r$ satisfying the relation

$$
\mathscr{Q}_{x-x_{e}^{1}}\left(Q^{-1}, d\right) \subseteq\left\{x \in \mathbb{R}^{2}: f^{i^{T}}\left(x-x_{e}^{1}\right) \leq \rho_{1}-u_{e}^{1}\right\}
$$

is an admissible domain of attraction of the equilibrium $x_{e}^{1}$. Such an admissible domain is obtained by setting $d=0.0572$.

We next consider the case of the equilibrium $x_{e}^{2}$ lying on the boundary line $g_{2}^{T} x=1$ where $g_{2}^{T}=[1.2-0.4]$. Since $g_{2}^{T} b=0$ but the line vector $g_{2}^{T}$ is not a left eigenvector of matrix $A$, there does not exist any gain matrix $f^{2^{T}}$ and real number $h_{22}$ such that $g_{2}^{T}\left(A+b f^{2^{T}}\right)=h_{22} g_{2}^{T}$. Therefore, there does not exist any control making the half space $g_{2}^{T} x \geq 1$ positively invariant. Thus, we search for a stabilizing control $u(t)=f^{2^{T}}\left(x(k)-x_{e}^{2}\right)+u_{e}^{1}$ making a cone

$$
\begin{gathered}
\mathscr{C}_{x-x_{e}^{2}}\left(G_{2}^{*}\right)=\left\{x \in \mathbb{R}^{2}: G_{2}^{*}\left(x-x_{e}^{2}\right) \geq 0\right\} \\
\mathscr{C}_{x-x_{e}^{2}}\left(G_{2}^{*}\right) \subset\left\{x \in \mathbb{R}^{2}: g_{2}^{T} x \geq 1\right\}
\end{gathered}
$$

positively invariant. Setting $z(k)=x(k)-x_{e}^{2}$, this problem is converted to a problem of determination of a gain vector $f^{i} \in \mathbb{R}^{2}$ making the the system $z(k+1)=(A+$ $\left.b f^{2^{T}}\right) z(k)$ asymptotically stable, the cone $\mathscr{C}\left(G_{2}^{*}\right)$ positively invariant and $\mathscr{C}\left(G_{2}^{*}\right) \subset$ $\mathscr{C}\left(-g_{2}^{T}\right)$. By applying a standard eigenvalue assignment approach we determine the gain vector

$$
f^{2^{T}}=[-2.06102,7537]
$$

for which matrix $A+b f^{2^{T}}$ has the eigenvalues $\lambda_{1}=0.4$ and $\lambda_{2}=0.7$ associated with the left eigenvectors

$$
\begin{aligned}
& v_{1}^{* T}=\left[\begin{array}{ll}
-0.5289 & 0,8487
\end{array}\right] \\
& v_{2}^{* T}=\left[\begin{array}{ll}
0.4580 & -0.8890
\end{array}\right]
\end{aligned}
$$

respectively. Then, setting

$$
G_{2}^{*}=\left[\begin{array}{l}
v_{1}^{* T} \\
v_{2}^{* T}
\end{array}\right]
$$

we get

$$
G_{2}^{*}\left(A+b f_{2}^{T}\right)=\operatorname{diag}\left(\lambda_{1}, \lambda_{2}\right) G_{2}^{*}
$$

and

$$
k^{T} G_{2}^{*}=g_{2}^{T}
$$

with

$$
k^{T}=[10.84039 .8990]
$$

This implies that the polyhedral proper cone $\mathscr{C}\left(G_{2}^{*}\right)$ is positively invariant w.r.t. the system $z(k+1)=\left(A+b f^{2^{T}}\right) z(k)$ and satisfies the set relation $\mathscr{C}\left(G_{2}^{*}\right) \subset \mathscr{C}\left(g_{2}^{T}\right)$. Therefore, with the control $u(k)=f^{2 T}\left(x(k)-x_{e}^{2}\right)+u_{e}^{2}$ the cone $\mathscr{C}_{x-x_{e}^{2}}\left(G_{2}^{*}\right)$ is positively invariant. 


$$
\mathscr{C}_{x-x_{e}^{2}}\left(G_{2}^{*}\right)=\left\{x \in \mathbb{R}^{2}: G_{2}^{*}\left(x-x_{e}^{2}\right) \geq 0\right\}
$$

It is a simple task to show that this is also an admissible domain of attraction

The forbidden region $\mathscr{P}(G, w)$, the positively invariant region $\mathscr{C}\left(g_{1}^{T}\right)$ and the admissible domains of attraction $\mathscr{D}_{1}$ and $\mathscr{D}_{2}$ of the closed-loop systems with controls $u^{(1)}$ and $u^{(2)}$ respectively are shown in Figure 3.

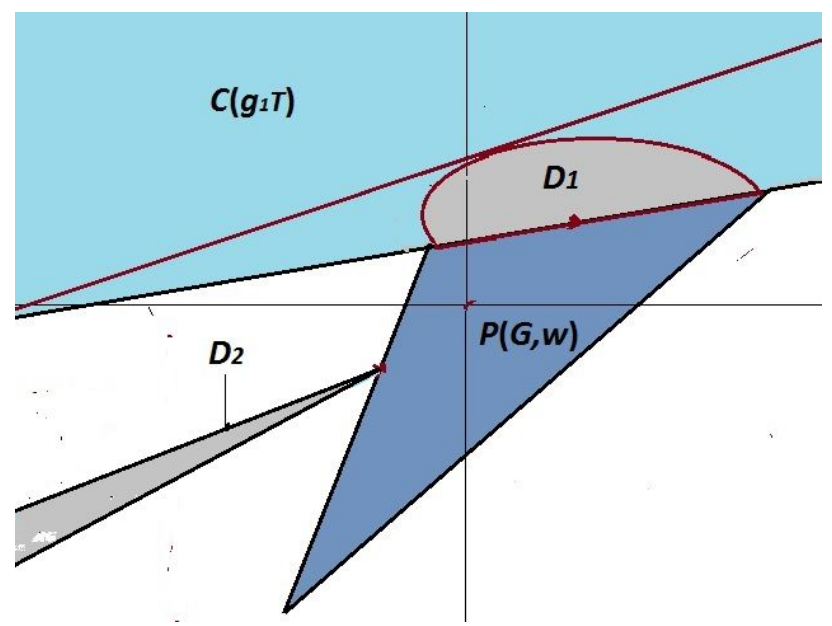

Fig. 3 The forbidden region $\mathscr{P}(G, w)$, the positively invariant region $\mathscr{C}\left(g_{1}^{T}\right)$ and the admissible domains of attraction $\mathscr{D}_{1}$ and $\mathscr{D}_{2}$ of the closed-loop systems with controls $u^{(1)}$ and $u^{(2)}$ respectively.

\section{Concluding remarks}

We have studied the Linear Constrained Regulation Problem around an equilibrium situated on the boundary of a polyhedral region where the state constraints are satisfied. It has been shown that, in this case, the stabilizability of the pair $(A, B)$ is not sufficient for the existence of an admissible linear state-feedback control because additional conditions of geometric nature are necessary to be satisfied. First, it has been shown that if a control law making the maximal admissible set a domain of attraction exists, then it may be determined by solving a linear programming problem. Next, the cases when such a control does not exist have been studied and appropriate design approaches based on convex optimization and/or eigenstructure assignment methods for determining stabilizing linear state-feedback controllers and corresponding admissible domains of attraction have been proposed. It should be noticed that the domains of attraction obtained by these approaches are not the maximal ones. For polyhedral domains however, they may be considered as the starting domains 
in the application of iterative approaches of enlargement of admissible domains of attraction [2], [3].

All the design techniques developed in this chapter are readily extendable to the case of continuous-time systems [6].

Acknowledgements The second author acknowledges the support of the grant CNCS - UEFISCDI, project number PN-III-P1-1.1-TE-2016-0862, MOSCBIOS, within PNCDI III.

\section{References}

1. Andry, A., Shapiro, E., and Chung, J. (1983). Eigenstructure assignment for linear systems. IEEE Transactions on Aerospace and Electronic Systems, 5, 711-729.

2. Athanasopoulos, N., and Bitsoris, G. (2009). A Novel Approach to the Computation of the Maximal Controlled Invariant Set for Constrained Linear Systems. Proceedings of the 10th European Control Conference Budapest, Hungary, (pp. 3124-3129).

3. Athanasopoulos, N., Bitsoris, G. and Lazar, M.(2014). Construction of invariant polytopic sets with specified complexity. Int. J. Control, 87(8), 1681-1693.

4. Benzaouia, A., and Burgat, C. (1988a). Regulator problem for linear discrete-time systems with nonsymmetrical constrained control. International Journal of Control, 48(6), 2441-2451.

5. Benzaouia, A., and Burgat, C. (1988b). The regulator problem for a class of linear systems with constrained control. Systems and Control Letters, 10, 357-363.

6. Bitsoris, G., Olaru, S., and Vassilaki, M. (2014). On the Linear Constrained Regulation Problem for Continuous-Time Systems. Proceedings of the 19th IFAC World Congress, Cape Town, South Africa. (pp. 24-29).

7. Bitsoris, G. (1986) Sur l'existence des ensembles invariants polyhedraux des systemes lineaires. Technical Report 86015, LAAS-CNRS, Toulouse, France.

8. Bitsoris, G.(1988). Positively invariant polyhedral sets for discrete-time systems. Int. J. Control, 47(6), 1713-1726.

9. Bitsoris, G. (1988). On the positive invariance of polyhedral sets for discrete-time systems. Systems and Control Letters, 11(3), 243-248.

10. Bitsoris, G., and Olaru, S. (2013). Further Results on the Linear Constrained Regulation Problem. Proceedings of the 21st IEEE Mediterranean Conference on Control and Automation, Platanias-Chania, Crete-Greece.

11. Bitsoris, G., and Truffet, L. (2011). Positive invariance, monotonicity and comparison of nonlinear systems. Systems \& Control Letters, 60(2), 960-966.

12. Bitsoris, G., G and Vassilaki, M. (1990). The linear constrained regulation problem for discrete-time systems. Proceedings of the 11th IFAC World Congress, Tallinn-Estonia, (pp. 287-292).

13. Bitsoris, G. and Vassilaki, M. (1993) Design techniques of linear constrained discrete-time control systems. In T.Leondes, editor, Control and Dynamic Systems. 56 (pp. 1-49). Academic Press, New York. Inc.

14. Bitsoris, G., and Vassilaki, M. (1995b). Constrained regulation of linear systems. Automatica, 31(2), 223-227.

15. Blanchini, F. and Miani, S. (2007). Set-Theoretic Methods in Control, Systems \& Control: Foundations \& Applications, Birkhauser, Boston-Basel-Berlin.

16. Blanchini, F. (1991). Constrained control for uncertain linear systems. J. Optimization Theory and applications, 71(3), 465-484.

17. Blanchini, F. and Ukovich, W. (1993). A linear programming approach to the control of discrete-time periodic system with state and control bounds in the presence of disturbance. Journal of Optimization Theory and Applications, 73(3), 523-539. 
18. Boyd, S., El Ghaoui, L., Feron, E., and Balakrishnan, V. (1994). Linear matrix inequalities in system and control theory. SIAM, Philadelphia-USA.

19. Burgat, C., Benzaouia, A., and Tarbouriech, S., (1990). Positively invariant sets of discretetime systems with constrained inputs. Int. J. Syst. Science. 21(7), 1249-1271.

20. Castelan, E. B., and Hennet, J.-C., (1992). Eigenstructure assignment for state constrained linear continuous time systems. Automatica 28(3), 605-611.

21. Gantmacher, F. R., (1960). The Theory of Matrices. Chelsea Pub. Co., New York.

22. Gilbert, E. and Tan, K. (1991). Linear systems with state and control constraints: The theory and the applications of the maximal output admissible sets. IEEE Transactions on Automatic Control, 36(9), 1008-1020.

23. Gutman, P. O., and Hagander, P. (1985). A new design of constrained controllers for linear systems. IEEE Transactions on Automatic Control, 30(1), 22-33.

24. Hennet, J.C. (1995). Discrete-time constrained systems, in: T.Leondes, editor, Control and Dynamic Systems. , Academic Press, Inc., 71, (pp. 157-213).

25. Molchanov, A.P., and Pyatinskii, E.S. (1986). Lyapunov functions that specify necessary and sufficient conditions of absolute stability of nonlinear control systems III. Automatika i Telemekhanika, 5(1), 38-49.

26. Prodan, I., Bitsoris, G., Olaru, S., Stoica, C., and Niculescu, S. (2013). Constrained Control Design for Linear Systems with Geometric Adversary Constraints., Proc. 5th IFAC Symposium on System Structure and Control, Grenoble-France. (pp. 815-820).

27. Rakovic, S.V., and Fiacchini, M. (2008). Invariant Approximation of the Maximal Invariant Set or "Encircling the Square". Proceedings of the 17th IFAC World Congress, Seoul, Korea, (pp. 6377-6382).

28. Srinathkumar, S., and Rhoten, R., (1975). Eigenvalue/eigenvector assignment for multivariable systems. Electronics Letters, 11(6), 124-125.

29. Ten Dam, A.A., and Nieuwenhuis, J.W. (1995). A linear programming algorithm for invariant polyhedral sets of discrete-time linear systems. Systems Control Letters, 25(5), 337-341.

30. Valcher, M.E., and Farina, L. (2000). An algebraic approach to the construction of polyhedral invariant cones. SIAM J. Matrix Anal. Applications, 22(2), 153-171.

31. Vassilaki, M., Hennet, J.C. and Bitsoris, G. (1988). Feedback control of linear discrete-time systems under state and control constraints. Int. J. Control, 47(6), 1727-1735. 